\title{
Ultrastructural Modifications in the Mitochondria of Hypoxia-Adapted Drosophila melanogaster
}

\author{
Guy Perkins ${ }^{19}$, Yu-hsin Hsiao ${ }^{2}$, Songyue Yin ${ }^{3}$, Jonathan Tjong ${ }^{1}$, My T. Tran ${ }^{1}$, Jenna Lau ${ }^{2}$, Jin Xue ${ }^{2}$, \\ Siqi Liu ${ }^{3}$, Mark H. Ellisman ${ }^{1}$, Dan Zhou ${ }^{2 * 9}$
}

1 National Center for Microscopy and Imaging Research, University of California San Diego, La Jolla, California, United States of America, 2 Department of Pediatrics, University of California San Diego, La Jolla, California, United States of America, 3 Beijing Institute of Genomics, Chinese Academy of Sciences, Beijing, P.R. China

\begin{abstract}
Chronic hypoxia $(\mathrm{CH})$ occurs under certain physiological or pathological conditions, including in people who reside at high altitude or suffer chronic cardiovascular or pulmonary diseases. As mitochondria are the predominant oxygen-consuming organelles to generate ATP through oxidative phosphorylation in cells, their responses, through structural or molecular modifications, to limited oxygen supply play an important role in the overall functional adaptation to hypoxia. Here, we report the adaptive mitochondrial ultrastructural modifications and the functional impacts in a recently generated hypoxiaadapted Drosophila melanogaster strain that survives severe, otherwise lethal, hypoxic conditions. Using electron tomography, we discovered increased mitochondrial volume density and cristae abundance, yet also cristae fragmentation and a unique honeycomb-like structure in the mitochondria of hypoxia-adapted flies. The homeostatic levels of adenylate and energy charge were similar between hypoxia-adapted and naïve control flies and the hypoxia-adapted flies remained active under severe hypoxia as quantified by negative geotaxis behavior. The equilibrium ATP level was lower in hypoxiaadapted flies than those of the naïve controls tested under severe hypoxia that inhibited the motion of control flies. Our results suggest that the structural rearrangement in the mitochondria of hypoxia-adapted flies may be an important adaptive mechanism that plays a critical role in preserving adenylate homeostasis and metabolism as well as muscle function under chronic hypoxic conditions.
\end{abstract}

Citation: Perkins G, Hsiao Y-h, Yin S, Tjong J, Tran MT, et al. (2012) Ultrastructural Modifications in the Mitochondria of Hypoxia-Adapted Drosophila melanogaster. PLoS ONE 7(9): e45344. doi:10.1371/journal.pone.0045344

Editor: Alicia J. Kowaltowski, Instituto de Química - Universidade de São Paulo, Brazil

Received October 28, 2011; Accepted August 21, 2012; Published September 19, 2012

Copyright: (c) 2012 Perkins et al. This is an open-access article distributed under the terms of the Creative Commons Attribution License, which permits unrestricted use, distribution, and reproduction in any medium, provided the original author and source are credited.

Funding: This study was supported by an American Heart Association award (0835188N) to DZ National Institutes of Health awards 5P41RR004050 and P41GM103412-24 to MHE. The funders had no role in study design, data collection and analysis, decision to publish, or preparation of the manuscript.

Competing Interests: The authors have declared that no competing interests exist.

*E-mail: d2zhou@ucsd.edu

9 These authors contributed equally to this work.

\section{Introduction}

Chronic hypoxia $(\mathrm{CH})$ is characterized by a constant, longlasting reduction in oxygen supply to tissues and cells. Such oxygen reduction occurs under certain physiological or pathological conditions, including in people who reside at high altitude or suffer chronic pulmonary diseases. One of the responses in organisms to $\mathrm{CH}$ is to decrease energy generation through marked suppression of adenosine $5{ }^{\prime}$-triphosphate (ATP) demand and supply pathways [1]. As mitochondria are the predominant organelles for oxygen utilization in the cell, their regulatory response to altered oxygen supply is proposed to play an important role in the overall functional adaptation to hypoxia. Indeed, previous studies have shown that mitochondrial metabolism is involved in $\mathrm{CH}$ adaptation via energy regulation, generation of reactive oxygen species (ROS), and apoptosis [2,3]. It is well established that mitochondria are remarkably plastic organelles that actively change their shape. Hypoxia-induced alterations of mitochondrial metabolism are thought to evoke changes in the mechanisms regulating mitochondrial structural dynamics. Such hypoxia-induced changes are widely considered as important pathogenic factors that contribute to hypoxic/ischemic cell injury in various organs including brain, heart, kidney and liver
$[4,5,6,7,8]$. Although hypoxia-induced alterations on the dynamics of mitochondrial network formation in the cell have been reported [9], to date, the detailed three-dimensional (3D) structural modifications within mitochondrion have not been investigated. As functional modifications in mitochondria may play important roles in protecting cells from hypoxic injury [10,11], it is important to determine the hypoxia-induced changes in mitochondrial ultrastructure and the impact on mitochondrial function.

As a well-studied organism, Drosophila melanogaster offers many useful features that are convenient for dissecting the mechanisms underlying different kinds of human diseases. These advantages include high degree of conservation in fundamental biological pathways between Drosophila and humans [12], a shorter generation time and life span, a large number of progeny, the availability of many well characterized genetic tools to manipulate gene function, and relatively well-known anatomy and phenotypes [13,14]. To date, various Drosophila lines have been successfully generated to analyze the function of different human disease genes including those responsible for developmental and neurological disorders, cancer, cardiovascular disease, and metabolic and storage diseases $[15,16,17,18,19]$. The extensive conservation of mitochondrial structure, composition, and function across species 
also offers a unique opportunity to expand our understanding of human mitochondrial biology and disease in fruit fly [20,21,22].

In order to dissect the mechanisms underlying hypoxia tolerance, we recently generated a hypoxia-tolerant Drosophila melanogaster strain through laboratory selection that survives severe, otherwise lethal, hypoxic conditions [23]. Using this unique animal line, we have identified genes and pathways that regulate this remarkable hypoxia tolerance phenotype which include the role of transcription suppressor hairy and the activation of the Notch pathway [24,25]. Furthermore, a synchronized global suppression of metabolic genes, including those encoding mitochondrial proteins, was discovered in the hypoxia-adapted (HA) flies [25]. Interestingly, metabolic profiles in the HA and normoxic control (NC) flies following acute hypoxia exposure revealed that HA flies had a higher efficiency of ATP production, lowered pyruvate carboxylase flux and greater usage of Complex I over Complex II respiration [26], demonstrating that mitochondrial adaptive adjustment, through the modifications of structure and enzyme activities, is one of the key factors regulating survival under chronic long-lasting hypoxic condition. In current study, we used HA thoracic muscle as a system to study the alterations in mitochondrial ultrastructure and the functional impact in this hypoxia-adapted organism.

\section{Results}

\section{Muscle Structure and Mitochondrial Volume Density in HA Thoracic Muscle}

Typical electron micrographs of thoracic muscle from control (NC) and hypoxia-selected flies (HA) are shown in Figure 1A and 1B. The myofiber architecture was unaltered in the HA muscle compared to the NC muscle. However, the myofiber size from HA flies $\left(2.7 \pm 0.3 \mu \mathrm{m}^{2}\right)$ was smaller than those of NCs $\left(4.2 \pm 0.7 \mu \mathrm{m}^{2}\right)$ $(\mathrm{p}<0.01)$ (Figure 1G). In contrast, an increased mitochondrial volume density (volume fraction) was observed in the HA flies $(34 \pm 1.5 \%)$ as compared to $\mathrm{NC}$ controls $(29 \pm 0.6 \%) \quad(\mathrm{p}<0.01)$ (Figure 1D). The smaller myofibers in HA partially produced empty space between these fibers.

\section{Altered Mitochondrial Ultrastructure in HA Thoracic Muscle}

Electron tomography was used to characterize in $3 \mathrm{D}$ the membrane architecture of $\mathrm{NC}$ and $\mathrm{HA}$ thoracic muscle mitochondria (movie S1 and S2). The NG mitochondria appeared typical for thoracic muscle with densely packed lamellar cristae (Figure 2A-2B). We found that $\mathrm{HA}$ mitochondria showed localized swollen regions devoid of cristae (Figure 2D-2F) and more notably, small regions where the cristae have been sliced or fractured almost uniformly in cross-section as if a microscopic knife had sliced perpendicular to the long axis of 6 or more adjacent cristae and then the ends annealed, i.e., the cristae membrane closed around the break (Figure 2G-2I). Rod-like dense cores were often observed between the ends of the fractured cristae running perpendicular to the cristae membranes. The dense core appears to be almost as an avenue with the cristae as spaced sentinels (Figure 2J, 2K); they are likely the residue from cristae fragmentation and are separated by a light (translucent) band from the annealed cristae membranes. We also noted that the lamellar cristae in hypoxic mitochondria were not as extensive (large) as those in the control and often exhibited fenestrations (Figure 2L). A greater percentage of cristae in the HA thoracic muscle mitochondria were similar to the highly branched and severely fenestrated crista shown in Figure $2 \mathrm{M}$ than those in the NC mitochondria. The cristae membrane surface area, normal-
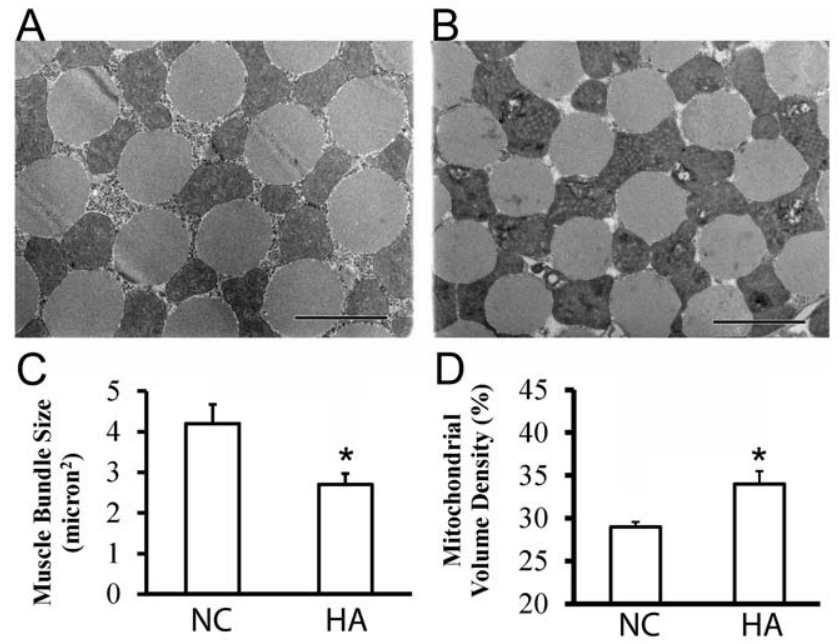

Figure 1. Muscle structure and mitochondrial volume density in HA thoracic muscle. (A) and (B): Typical electron micrographs of thoracic muscle from control (NC) and hypoxia-selected flies (HA) (scale $=1 \mu \mathrm{m}$ ). No significant modifications on myofiber architecture were determined in the HA muscle as compared to that of NC control. However, a reduced myofiber size (C) $\left(n=69,{ }^{*} p<0.01\right)$ and an increased mitochondrial volume density (volume fraction) (D) $(n=11$, ${ }^{*} \mathrm{p}<0.01$ ) were found in the thoracic muscle of HA flies. doi:10.1371/journal.pone.0045344.g001

ized to the mitochondrial outer membrane surface area, was significantly greater in the hypoxic thoracic muscle (Figure $2 \mathrm{~N}$ ) as was the cristae number, normalized to the mitochondrial crosssectional area (Figure 2O) compared with the NG mitochondria. The increase in cristae number reflects the cristae fragmentation noted and the increase in cristae membrane may be an adaptation to compensate for this fragmentation. The cristae width was found to differ between the longer cristae and the fragmented (and annealed) cristae in the HA mitochondria. The fragmented cristae in the HA mitochondria had a mean width of $31 \pm 0.5 \mathrm{~nm}$ (mean \pm SEM, $n=100$ ), whereas the longer cristae in the same mitochondria had a mean width of $23 \pm 0.3 \mathrm{~nm} \quad(\mathrm{n}=100)$, a difference highly statistically significant $(\mathrm{p}<0.001)$, and nearly identical to the width of $24 \pm 0.4 \mathrm{~nm}(\mathrm{n}=100)$ measured for the NC mitochondria.

A honeycomb-like pattern of light, roughly circular regions with a dense center was commonly observed in HA muscle mitochondria when the tomographic reconstruction was sliced in crosssection to the myofibrils (Figure $3 \mathrm{~A}$ ). The dense center is the cross section of the rod-like core. The segmented honeycomb-like pattern shows the spatial distribution and three-dimensional (3D) extent of these structures (Figure 3B-3E). These rods do not extend completely through the volume of the mitochondria, consistent with the views of their limited extent shown in Figure 2. Measurements were made from electron micrographs to determine the prevalence of this honeycomb-like pattern. Only $1 \%$ of the NG mitochondria showed the honeycomb-like pattern $(\mathrm{n}=175)$, whereas $49 \%$ of the HA mitochondria had this pattern $(\mathrm{n}=380)$. Other HA mitochondria appeared normal.

\section{Equilibrium Adenylate Contents and Hypoxia-induced Changes in ATP Level in the Thoracic Muscle of NC and HA Flies}

The equilibrium level of adenylate (i.e., ATP, ADP and AMP) in thoracic muscle was determined by HPLC. Besides a slight but significant difference in ADP content, ATP, AMP and energy 

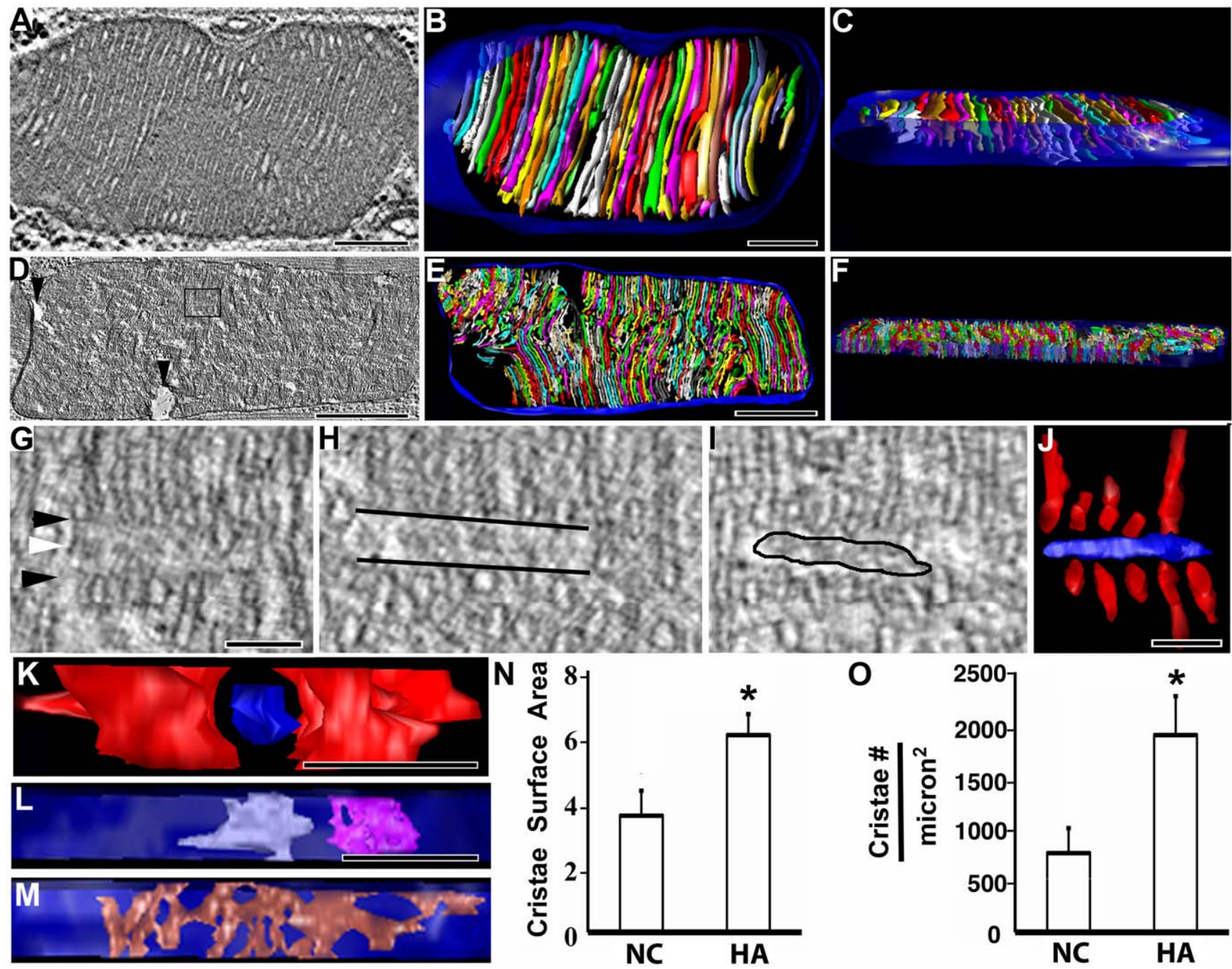

Figure 2. Ultrastructural modifications in the mitochondria of HA flies. (A) Slice through a tomographic volume of a normoxic mitochondrion found in thoracic muscle sliced longitudinally. Normal morphology is observed, including stacks of ordered cristae with light intracristal space and darker matrix. Scale $=200 \mathrm{~nm}$. (B) Segmented volume of the mitochondrial membrane structure. The cristae (various colors) were all found to be lamellar in form, as expected. This mitochondrial volume contained 42 cristae. Scale $=200 \mathrm{~nm}$. (C) Oblique view of the mitochondrion viewed partially through the outer membrane (blue) made translucent to observe the cristae arrangement. Same scale as B. (D) Slice through a tomographic volume of hypoxic thoracic muscle. This large mitochondrion displays a few regions devoid of cristae (arrowheads) and more notably, small regions where the cristae have been fractured (boxed example and shown expanded in G-I). Scale $=1000 \mathrm{~nm}$. (E) Top view of the membrane segmentation of the volume. This mitochondrial volume contained 641 cristae. Scale $=1000 \mathrm{~nm}$. (F) Oblique view of the mitochondrion viewed partially through the outer membrane to observe the cristae motif. Same scale as E. (G) Expanded view of the boxed region in D. This area and others in $(\mathrm{H})$ and $(\mathrm{I})$ from different slices of the volume show views of rod-like dense cores between fractured cristae. The edge of the rod is the fracture curve of the cristae (black arrowheads) where the cristae in a localized region fractured almost uniformly (black lines in $\mathrm{H}$ ) and then the ends annealed, i.e., the cristae membrane closed around the break. The dense core (white arrowhead) appears to be the degenerated cristae (black outline in I) that are separated by a white (translucent) band from the annealed cristae membrane curve. Scale $=100 \mathrm{~nm}$. (J) Top view of the segmented region of I showing the dense core (blue) surrounded by the cristae fragments (red). Scale $=100 \mathrm{~nm}$. (K) Side view perpendicular to the view in J, down the axis of the dense core appearing almost as an avenue with the cristae as spaced sentinels. Scale $=100 \mathrm{~nm}$. (L) Examples of two lamellar cristae in the hypoxic mitochondrion of panel $D$. These cristae were not as extensive as those in the control and often exhibited fenestrations (arrowhead). A greater percentage of cristae were similar to the highly branched and severely fenestrated crista shown in $(M)(* p<0.01)$. Scale $=500 \mathrm{~nm}$. (N) The cristae surface area, normalized to the mitochondrial outer membrane surface area, was significantly greater in the hypoxic thoracic muscle as was the cristae number, normalized to the mitochondrial cross-sectional area $(0)\left({ }^{*} \mathrm{p}<0.01\right)$. doi:10.1371/journal.pone.0045344.g002

charge (EG) showed no difference between $\mathrm{HA}$ and $\mathrm{NG}$ flies demonstrating that energy homeostasis is identical in the control $\mathrm{NG}$ flies living at room air condition and $\mathrm{HA}$ flies living at $4 \mathrm{kPa}$ $\mathrm{O}_{2}$ hypoxic condition (Figure $4 \mathrm{~A}$ and $4 \mathrm{~B}$ ). However, when exposed to a further lowered oxygen level (i.e., $2 \mathrm{kPa} \mathrm{O}_{2}$ ), $\mathrm{NC}$ flies exhibited a transient decline of ATP within the first 2 hours of exposure. This decline was recovered following continued exposure (Figure 4G). In contrast, HA flies showed a decline of ATP level in the first 2 hours and stayed at this lowered level in the following period of hypoxia exposure, suggesting that a new ATP homeostasis was created in the HA flies in this new oxygen environment (Figure 4C). Using electron microscopy, we measured the mitochondrial volume density of NC flies placed in $2 \mathrm{kPa} \mathrm{O}_{2}$ for 2 and 6 hours and compared with room-air NC flies 


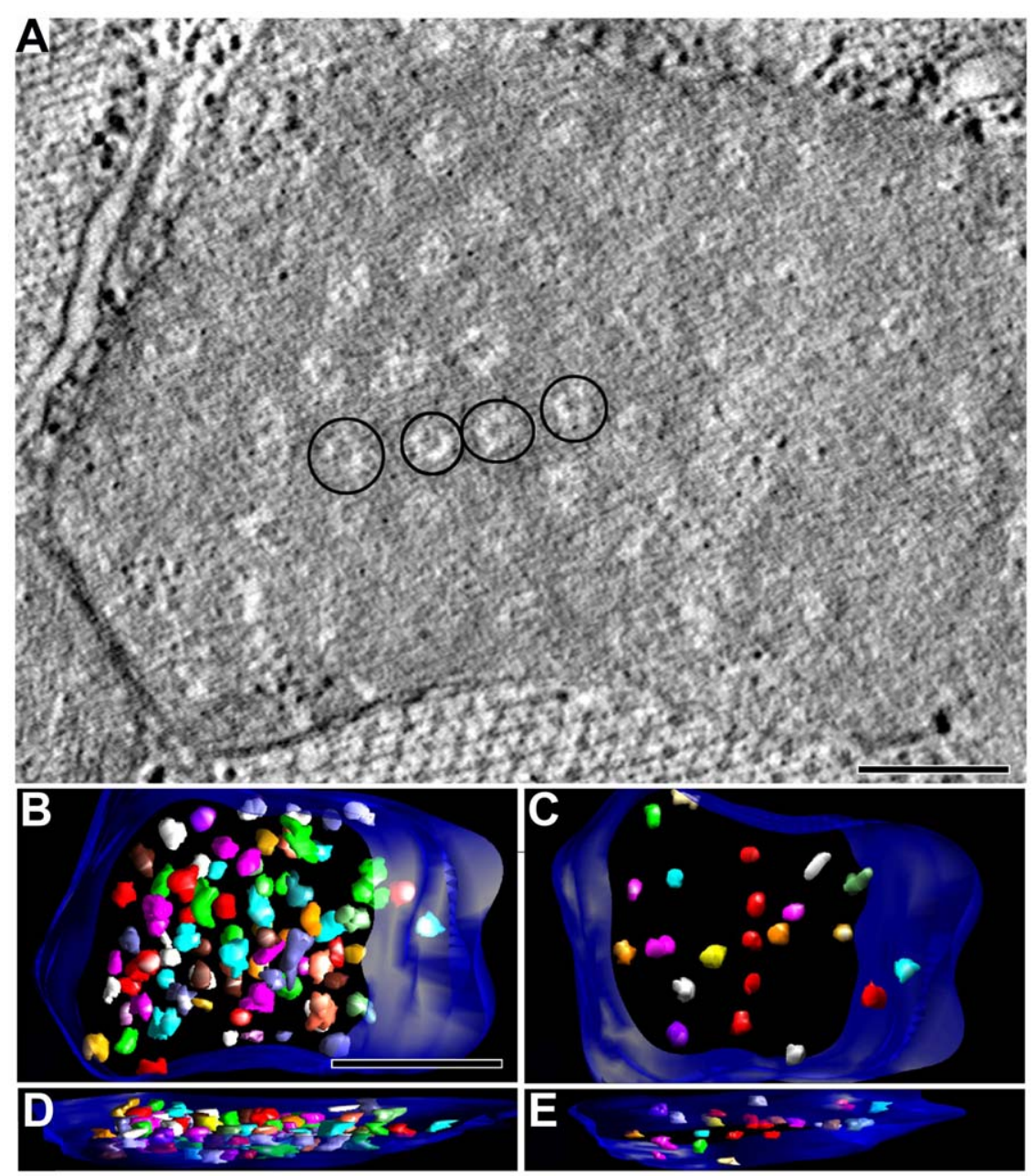

Figure 3. Honeycomb-like structures in the mitochondria of HA flies. (A) Slice through another tomographic reconstruction of a hypoxic mitochondrion in thoracic muscle oriented in cross-section (transverse) to the myofibrils. A honeycomb-like pattern of light, roughly circular regions with a dense center (4 circled) was found. Scale $=200 \mathrm{~nm}$. (B-E) The segmented honeycomb-like pattern (various colors) shows the spatial distribution and 3D extent of these structures. The full complement of rod-like structures having a dense core that is also rod-shaped, yet narrower is shown in B (top view) and C (oblique view). This mitochondrial volume contained 84 rods in the honeycomb-like structures. A subset of rods ( 23 total) is shown in $\mathrm{D}$ (top) and $\mathrm{E}$ (oblique) to note that these rods do not extend completely through the volume (height) of the mitochondrion. Scale $=500 \mathrm{~nm}$.

doi:10.1371/journal.pone.0045344.g003

and found no difference, consistent with the ATP measurements (MitoVolDensity 0 hrs: $31 \pm 2 \%, \mathrm{n}=9$; MitoVolDensity 2 hrs: $29 \pm 1 \%, \mathrm{n}=11$; MitoVolDensity 6 hrs: $32 \pm 1 \%, \mathrm{n}=8$; mean $\pm^{\prime \prime \prime}$ SEM).

To address whether the drop in ATP levels at the earliest time point ( 1 hour) shown in Figure 4 could be explained by a structural phenotype, we examined electron micrographs and quantified observed structural modifications (Figure S1). We found that the $\mathrm{NC}$ and HA flies exposed to hypoxia for 1 hour did not have the mitochondrial honeycomb-like pattern nor a change in mitochondrial volume density, but instead showed subregions of deformed and swollen cristae within a subset of mitochondria $(29 \pm 3 \%$ for $\mathrm{NC}, 14 \pm 3 \%$ for HA; mean $\pm \mathrm{SEM}$ ), consistent with the lowered levels of ATP at 1 hour. To separate whether the observed structural phenotype was influenced more by the genotype or by the environment, we interrogated whether that HA flies transferred to room oxygen level resemble the NG flies at this level. As with the NC and HA flies exposed to hypoxia for 1 hour, the HA flies raised in room air for one and two generations did not show a honeycomb-like pattern. We found that the mitochondrial volume density was significantly higher with the HA flies at room air for the first generation compared to the NC flies (38\% HA vs. $30 \% \mathrm{NC} ; \mathrm{p}<0.01$ ) (Figure $\mathrm{Sl}$ ). However, by the second generation, there was no statistical difference $(34 \%$ HA vs. $30 \%$ $\mathrm{NC} ; \mathrm{p}=0.13$ ). Moreover, the percent mitochondria with deformed cristae dropped from $12 \%$ (first generation) to $4 \%$ (second generation) compared to a baseline incidence of $5 \%$ for the NG. This suggests a quick reversion of the $\mathrm{HA}$ to the NC phenotype.

Negative Geotaxis Behavior of HA and NC Flies in Room Air and Hypoxic Condition

The geotaxis behavior of HA and NC flies in a test for climbing ability was investigated by recording the time taken for half of a group of 10 male flies to climb the $5 \mathrm{~cm}$ wall of a glass tube under room air or hypoxic conditions; each group of flies was tested three times in succession. The mean journey time for both hypoxia-selected and control flies was $<2$ seconds under room air condition with no significant differences. In contrast, in the hypoxic environment, the climbing time was significantly in- 


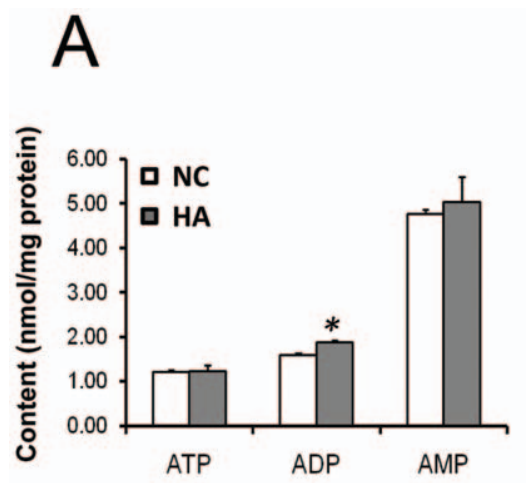

\section{B}
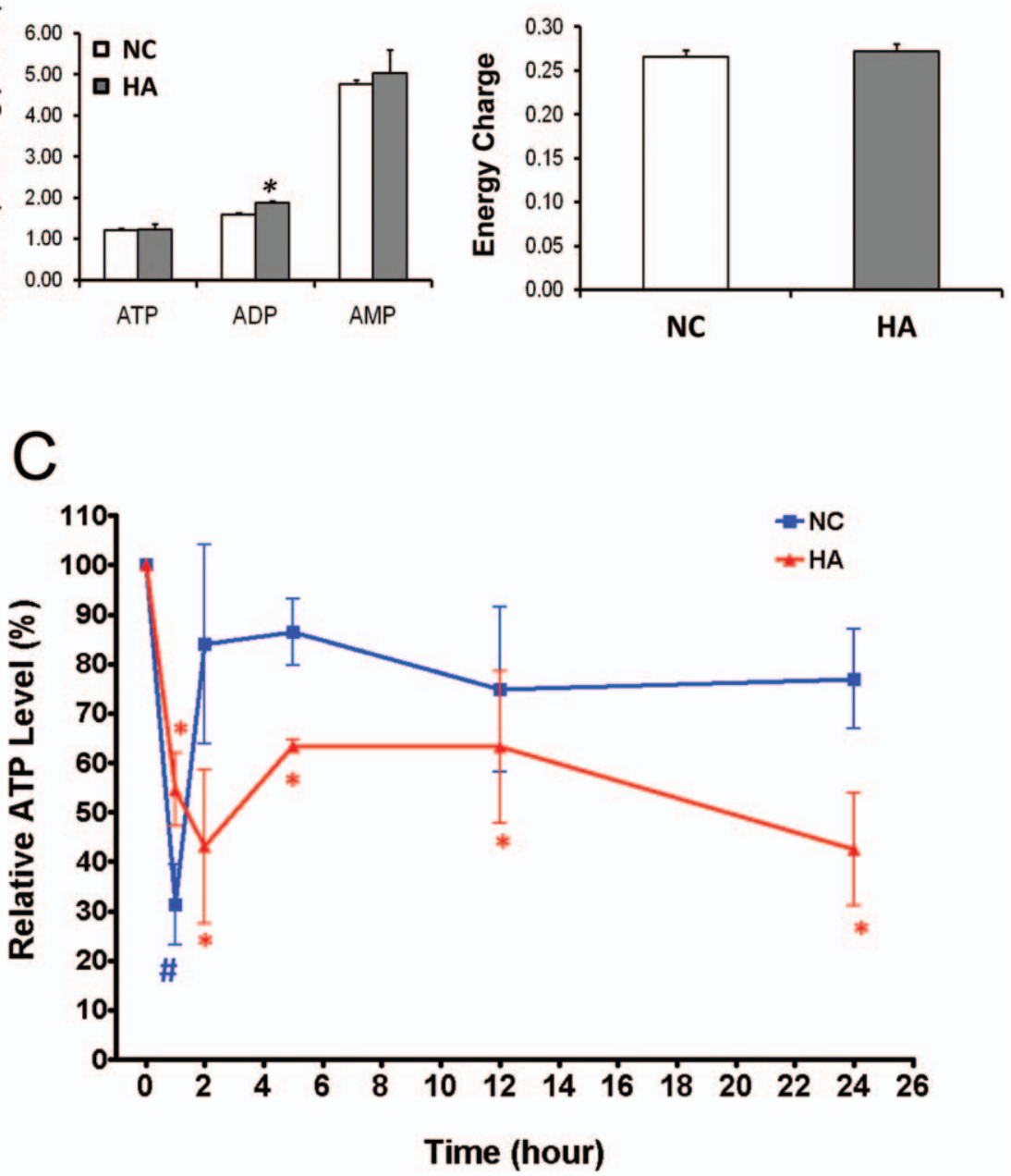

Figure 4. Equilibrium adenylate contents and hypoxia-induced changes in ATP level in the thoracic muscle of NC and HA flies. The equilibrium level of adenylates (i.e., ATP, ADP and AMP) in thoracic muscle was determined by HPLC. (A) No significant differences in ATP and AMP levels were determined between the $\mathrm{HA}$ flies living at $4 \mathrm{kPa} \mathrm{O}$ environment and $\mathrm{NC}$ controls living under room air condition $\left(21 \mathrm{kPa} \mathrm{O}_{2}\right)(\mathrm{n}=3$; $p>0.05)$. However, a slight but significant difference in ADP content was found in the HA flies $(n=3 ; * p<0.05)$. (B) EC showed no difference between HA and NC flies ( $n=3 ; p>0.05$ ). (C) Dynamic changes in ATP level under hypoxia in HA and NC thoracic muscle. A time course of changes in ATP level was measured in the thoracic muscle of $\mathrm{HA}$ and $\mathrm{NC}$ flies under hypoxic condition containing $2 \mathrm{kPa} \mathrm{O}_{2}$ during 24 hours. NC flies exhibited a transient decline of ATP within the first 2 hours of exposure. This decline was recovered following continued exposure (blue). HA flies also showed a decline of ATP level in the first 2 hours and stayed at this lowered level in the following period of hypoxia exposure time $(r e d)\left(n=6 ;{ }^{*}\right.$ and $\left.{ }^{*} p<0.05\right)$. doi:10.1371/journal.pone.0045344.g004

creased in the hypoxia-selected flies to $16.4 \pm 1.3$ seconds (mean \pm SEM) (Table 1). However, the geotaxis behavior was totally abolished in the NG control flies, which showed little upward movement within 10 minutes after tapped to the bottom. Furthermore, the total locomotion activity was dramatically reduced in the NG flies. Most of the NG flies ceased movement even with physical disturbances of the tube by shaking or tapping. In contrast, the HA flies exhibited normal regular motion activities during the time exposed to the hypoxic environment (see Movie S3, S4, S5, S6).

\section{Increased Sensitivity to Oxidative Stress in HA Flies}

As mitochondria regulate not only energy metabolism through oxidative phosphorylation but also oxidative injury through ROS generation, the mitochondrial structural rearrangement observed in the hypoxia-selected flies suggests that these flies might have different ROS generation rate and, therefore lifespan when treated with ROS generation reagents. To test this hypothesis, we determined the lifespan of hypoxia-selected and control flies with paraquat treatment. Paraquat is a widely used superoxide generator that stimulates oxidative stress when catalyzed in mitochondria $[27,28]$. We found that under the same treatment condition, the HA flies (both male and female) were more sensitivity to paraquat with a significantly shortened lifespan than controls as measured by a 50\% survival rate. As shown in Figure 5, with $5 \mathrm{mM}$ paraquat treatment, the $50 \%$ survival rate of male HA flies was significantly shortened $(4.9 \pm 1.1$ days) as compared to that of $\mathrm{NG}$ controls $(9.7 \pm 3.0$ days $)(\mathrm{p}<0.01)$. Similar results were obtained for female flies (data not shown). The 50\% survival rate was also shorter in $\mathrm{HA}$ than $\mathrm{NC}$ when treated with $10 \mathrm{mM}$ paraquat (data not shown). 
Table 1. Medium journey time for hypoxia-selected and control flies to climb $2.5 \mathrm{~cm}$ distance in room air and $2 \mathrm{kPa} \mathrm{O}_{2}$ hypoxic environment.

\begin{tabular}{|c|c|c|}
\hline & Room Air (second) & $2 \mathrm{kPa} \mathrm{O}_{2}$ Hypoxia (second) \\
\hline Normoxia control flies $(n=90)$ & $<2$ & $>600$ \\
\hline Hypoxia-selected flies $(n=90)$ & $<2$ & $16.4 \pm 1.3$ \\
\hline
\end{tabular}

It has been shown that a hypoxic environment may affect the food preference and intake of flies [29]. To determine the influence of food intake on paraquat treatment in the NC and HA flies, we measured food intake using a capillary feeding assay under room air condition, the same condition under which the paraquat treatment was performed. We found that the food intake was similar between $\mathrm{HA}(0.54 \pm 0.02 \mu \mathrm{l} / \mathrm{mg}$ body weight/day $)$ and NC flies $(0.49 \pm 0.02 \mu \mathrm{l} / \mathrm{mg}$ body weight/day) (mean \pm SEM; $\mathrm{n}=9, \mathrm{p}>0.05)$, suggesting that $\mathrm{NG}$ and $\mathrm{HA}$ flies received a comparable dosage of paraquat during the treatment.

\section{Discussion}

Muscle is an oxidative tissue of the body in which the production of cellular energy, in the form of adenosine triphosphate (ATP), occurs primarily via the process of oxidative phosphorylation in mitochondria. In order to sustain normal cellular function, mitochondria require a constant supply of fuels and oxygen. When oxygen delivery is impaired through hypoxia (such as decreased oxygen carrying capacity or decreased convective transport or in healthy individuals at altitude), a process of adaptation must occur to maintain cellular energy homeostasis. Indeed, it has been proposed that hypoxia is a key inducer of adaptation in skeletal muscle at altitude and with physical training [30,31]. However, mechanisms underlying mitochondrial adaptation in a hypoxic environment are still largely uncertain. In this study, using a hypoxia-adapted Drosophila melanogaster line [23], we investigated the effects of $\mathrm{CH}$ on muscle and mitochondrial

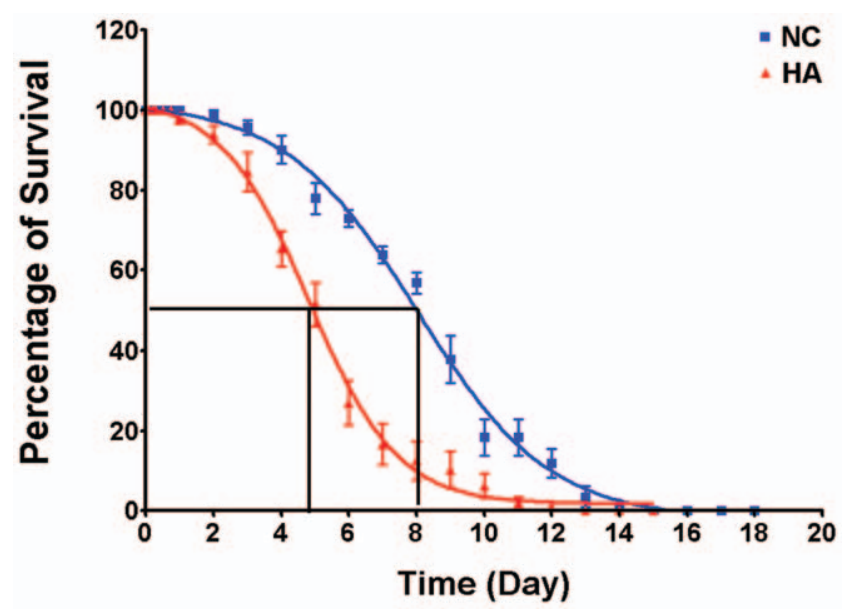

Figure 5. Increased sensitivity to oxidative stress in HA flies. A significantly shortened lifespan was determined in the HA flies (red) as compared to $\mathrm{NC}$ controls (blue) with $5 \mathrm{mM}$ paraquat treatment under room air condition. The $50 \%$ survival of $\mathrm{HA}$ was $4.9 \pm 1.1$ days, and the $50 \%$ survival of NC controls was $9.7 \pm 3.0$ days (summarized from 6 separate experiments, $p<0.01$ ).

doi:10.1371/journal.pone.0045344.g005 structure, muscle function, ATP metabolism and response to oxidative stress.

In the HA flies, we observed a reduction of myofiber size (i.e., $36 \%, \mathrm{p}<0.01)$ and a slight, but significant, increase in mitochondrial volume density (i.e., $7 \%, \mathrm{p}<0.01$ ) (Figure 1). Interestingly, the reduction in muscle fiber size has also been observed in humans and animals that were exposed to chronic hypoxia including permanent high altitude residents and lowlanders or rodents that were exposed to hypoxia for a prolonged period of time [32,33], suggesting that the mechanisms underlying $\mathrm{CH}$ induced changes in muscle is conserved between vertebrates and invertebrates. It is believed that, on one hand, the reduced fiber size demonstrated a down-regulation of protein synthesis, which could significantly reduce energy demand. And, on the other hand, the reduced fiber size could potentially improve the efficiency of oxygen supply through vascular circulation in mammals or tracheal delivery in fruit fly. In terms of mitochondrial volume density, loss of skeletal muscle mitochondrial density is a consistent finding in humans at altitude and patients with respiratory disease $[34,35]$. For example, a reduction in mitochondrial volume density was reported in $\mathrm{CH}$ exposed human subjects including individuals from the Tibetan population $[35,36]$. Such reduction in mitochondrial volume density was retained even in the second-generation who were born and raised at a much lowered altitude [32], suggesting that the reduced mitochondrial volume density is a heritable trait in this high altitude adapted Tibetan population. However, a muscle-type dependent, $\mathrm{CH}$-induced change in mitochondrial volume density has been found in rats treated with $\mathrm{CH}$ where the mitochondrial volume density was increased in the soleus muscle but decreased in the extensor digitorum longus muscle in the same animal treated with $\mathrm{CH}$ [33]. The slight, but significant, increase in mitochondrial volume density detected in the HA flies suggested that change in mitochondrial volume density might be an adaptive mechanism that potentially maximizes the efficiency of aerobic respiration under oxygen limited conditions.

Electron microscope tomography allowed us to determine detailed 3D mitochondrial structural modifications in the HA flies. In the thoracic muscle of HA flies, we found localized cristae fragmentation and matrix swelling (Figure 2), but to a lesser extent than reported in other studies where mitochondria enlargement, cristae rupture and loss were commonly described [37,38,39,40]. Instead of unrepaired cristae rupture, we found that the ends of the fragmented cristae membranes had rejoined (annealed) that appeared to be a repair mechanism. This annealing produced cristae with larger widths. These wider cristae may be a natural consequence of local changes in osmolarity because of mixing of the intracristal and matrix compartments upon rupture and subsequent annealing, consistent with studies of cristae width changes influenced by osmolarity [41]. The wider cristae may also reflect an energy minimum of the bilayer membrane based on a thermodynamic model of curvature [42], meaning that the 
combination of the shorter cristae and the curvature of their ends produces wider cristae to satisfy an energy minimum.

Cristae fragmentation and matrix swelling have been reported through a variety of perturbations $[43,44,45,46]$ including myocardial ischemia $[47,48]$. The cristae fragmentation observed in our longitudinal sections produced an interesting honeycomblike pattern in cross-section that was not observed in $\mathrm{NC}$ mitochondria (Figure 3). Interestingly, similar honeycomb-like cristae structures have been reported in various previous studies. For example, it has been observed in a Drosophila line carrying pathogenic ATP6 mutation, which showed a honeycomb appearance consisting of numerous cristae vesicles that were often interconnected with each other [49]. It is noteworthy that this group also reported fenestrated cristae in the mutant samples that we found to be a hallmark of HA mitochondria. In addition, in Barth syndrome patients [50] and a mouse model of the disease [51], a honeycomb structure was also observed inside mitochondria. Another similar pattern was reported in TIM44-transfected COS7 cells [52]. In all of these instances, however, the honeycomb patterns were different than what we observed and appeared to be paracrystalline in the mitochondria described by Acehan and coworkers and Wada and Kanwar. These authors noted that this pattern could conceivably be derived from mitochondria under stress conditions, including early stages of ischemia. It seems natural to us to also suggest that the cristae fragmentation, fenestration, and the novel honeycomb-like pattern observed in HA mitochondria is due to the stress of hypoxia. However, we cannot exclude the contribution of genetic variation in the HA flies derived from hypoxia selection. Compensation for hypoxic stress might be manifested by the increase in cristae membrane abundance and thus ATP-producing capacity. The increased cristae fragmentation measured in HA mitochondria is consistent with the report that oxygen deficiency increased the cristae content of mitochondria in myocardial cells [53]. Taken altogether, these findings, including ours, demonstrate that morphological plasticity contributes to mitochondrial adaptation under hypoxic environmental constraint.

Maintaining an effective ATP level is essential for muscle function. Since both cross-bridge cycling at the sarcomere during contraction and calcium reuptake to the sarcoplasmic reticulum during relaxation are heavily dependent on ATP hydrolysis, a compromise in cellular energy can lead to more rapid fatigue in exercising muscle. For example, in cardiac muscle, energetic impairment has been associated with the pathogenesis of hypertrophic cardiomyopathy and sudden cardiac death [54]. We detected similar levels of adenylates and energy charge between $\mathrm{HA}$ (living in $4 \mathrm{kPaO} \mathrm{O}_{2}$ severe hypoxic environment) and $\mathrm{NC}$ (living at $21 \mathrm{kPa} \mathrm{O}$ room air condition) with a slightly higher level of ADP in the muscle samples of HA flies (Figure 4A-B), demonstrating that the adenylate homeostasis is maintained well in this hypoxia-adapted Drosophila lines at their "native" hypoxic environment. As a previous metabolomic study in the same Drosophila line did not find evidence supporting a significant upregulation of anaerobic metabolic activities in the HA flies [26], such well-maintained adenylate homeostasis is very likely through aerobic metabolism in well-functioning mitochondria. Indeed, the same study suggested that HA flies produced more ATP per glucose and created fewer protons than control flies. And HA flies may have lower rates of glycolysis, less acidosis, and more efficient use of substrate during acute hypoxic stress [26]. This notion is further supported by the observation that, under hypoxic condition (i.e., $2 \mathrm{kPa} \mathrm{O}_{2}$ ), HA flies showed normal, even though delayed, geotaxis behavior. However, in NG flies, such geotaxis activity was totally abolished (Table 1), suggesting that the HA muscle was well functioning in the severe hypoxic environment.

The dynamic change in ATP level following acute hypoxia treatment (i.e., $2 \mathrm{kPa} \mathrm{O}_{2}$ ) was measured during a 24 hours exposure time. The NC flies showed a rapid drop in the first hour and an almost full recovery at the 2 hour time point in this hypoxic environment. In contrast, the HA flies exhibited a slower drop in the first 2 hours followed by a partial recovery and remained at a lowered ATP level ( $\sim 60 \%$ of the original level) for the rest of the experimental time (Figure 4C). Such a lowered ATP level might result from higher physical and cellular activities in HA flies as compared to NC controls under hypoxic condition. As mentioned above, the geotaxis activity was well retained in the HA flies as measured after 2 hours treatment with $2 \mathrm{kPa} \mathrm{O}_{2}$. In contrast, following the same treatment time, even though the ATP level was recovered to normal value, the majority of $\mathrm{NC}$ flies remained motionless with totally abolished geotaxis activities in this hypoxic environment (Table 1), which may also indicate that the efficiency of muscle energetic regulation is different between $\mathrm{HA}$ and $\mathrm{NC}$ flies. Indeed, it has been reported that chronic hypoxia improves energy supply regulation in the cardiac muscle of mice [55]. As shown in Figure 2, HA flies generated fragmented cristae and a larger cristae surface area in the mitochondria during development in their "native" chronic hypoxic environment. Such increased cristae fragmentation and cristae abundance in the HA mitochondria may play a critical role in increasing energy supply by providing an enlarged reaction surface and efficient proton flow under low oxygen condition. Consequently, this adaptive structural modification preserved the muscle function and the physical activity of HA flies in hypoxia.

It has been found that $\mathrm{CH}$ induces oxidative stress in muscle. For example, climbers who have been exposed for prolonged periods to extreme altitude exhibit evidence of oxidative stress in skeletal muscles [56,57]. Similar oxidative changes in muscle biopsies have also been observed in hypoxic chronic obstructive pulmonary disease (COPD) patients but not in obstructed COPD patients who do not exhibit hypoxia [58]. Although the mechanisms for hypoxia-induced ROS formation in skeletal muscle, or in any other tissue, are still not fully understood, it has been proposed that the mitochondrion is the strongest candidate for the source of hypoxia-induced ROS formation [59,60,61]. Using paraquat (1,1'-dimethyl-4,4'-bipyridinium dichloride), a ROS generator through mitochondria metabolism, we compared the susceptibility to oxidative stress between HA and $\mathrm{NC}$ flies. We found that HA flies are more vulnerable to paraquat treatment with a significantly shortened lifespan than NG when treated with the same dosage (Figure 5). Paraquat has been widely used to stimulate superoxide production in organisms and cells. Previous studies have shown that superoxide production by paraquat occurs in the mitochondrial matrix where it was reduced by complex I to form the paraquat radical cation that then reacted with oxygen to form superoxide $[27,28]$. Such superoxide production can cause extensive mitochondrial oxidative damage $[62,63,64,65]$. Our previous studies have found that the activity of respiratory complex I was elevated in HA flies [26,66]. An increased complex I activity may increase the level of paraquatderived ROS production. Furthermore, it has been shown that the ROS level and its defense mechanisms were reduced in flies living in chronic constant hypoxic condition [66,67]. Therefore, the increased sensitivity to paraquat treatment in HA flies might be a combined result from both an increased production of paraquatderived ROS and a reduced ROS defense.

In summary, we observed significant ultrastructural modifications with increased cristae fragmentation and abundance in the 
mitochondria of thoracic muscle from hypoxia-adapted Drosophila melanogaster. Our results suggest that such morphological rearrangement may potentially be an important adaptive mechanism that plays a critical role in preserving adenylate homeostasis and metabolism as well as muscle function under chronic hypoxic conditions.

\section{Materials and Methods}

\section{Drosophila Melanogaster Stocks and Culture}

The parental D. melanogaster lines, HA and control flies used in this study were described previously. Briefly, 27 isogenic Drosophila melanogaster lines were balance-pooled to generate the parental population for experimental selection of hypoxia-adapted flies and controls. These 27 isogenic lines had different recovery times from acute anoxia stupor and eclosion rates under hypoxic conditions, demonstrating genetic diversity in terms of hypoxia response [23]. The hypoxia-selected (HA) and control (NC) flies were used for the current study. The flies were cultured at room temperature on standard cornmeal/agar medium. The HA flies were cultured under the hypoxic condition containing $4 \mathrm{kPa} \mathrm{O}_{2}$ balanced with nitrogen. And the NG flies were cultured under room air $(21 \mathrm{kPa}$ $\mathrm{O}_{2}$ ) condition.

\section{Negative Geotaxis Assay}

The climbing test was performed according to a previous description with modifications [68]. A group of 10 male flies (5-7 days old) were placed in a 15 -ml glass tube with food and were gently tapped to the bottom of the tube. The time taken for half of the knocked-down flies to climb $2.5 \mathrm{~cm}$ of the tube wall was recorded. Each group was tested 10 times in room air $\left(21 \mathrm{kPa} \mathrm{O}_{2}\right)$ or hypoxic condition $\left(2 \mathrm{kPa} \mathrm{O}_{2}\right)$ in a computer-controlled atmosphere chamber (Model 8301055; Coy laboratory Products Inc, Grass Lake, MI).

\section{Measurement of Adenylate Contents and ATP Homeostasis}

Adult flies were rapidly frozen using liquid nitrogen. Thoracic muscle from 40 50 flies was isolated on dry-ice and homogenized in $300 \mu \mathrm{l}$ pre-chilled 2.3 M Perchloric acid (PCA) to extract free nucleotides. The homogenate was placed on ice for $15 \mathrm{~min}$ and then centrifuged at $13,000 \mathrm{~g}$ for $5 \mathrm{~min}$. $1 \mathrm{M} \mathrm{K}_{2} \mathrm{CO}_{3}$ was added to the supernatant until the $\mathrm{pH}$ was neutralized. After centrifugation, the supernatant was filtered with a $0.22 \mu \mathrm{m}$ membrane and stored in a $-20^{\circ} \mathrm{C}$ freezer until use for HPLC analysis [69].

The HPLC system (Shimadzu, Japan) consists of a Shimadzu CBM-20A controller, a SPD-20A variable wavelength UV detector, a LC-6AD pump and a Phenomenex C18 column $(25 \mathrm{~cm} \times 4.6 \mathrm{~cm} ; 5 \mu \mathrm{m}, 100 \mathrm{~A})$. The nucleotides extracted from the thoracic muscle $(100 \mu \mathrm{l})$ were run in a HPLC program to separate nucleotides (100\% A solution $\left(30 \mathrm{mM} \mathrm{KH}_{2} \mathrm{PO}_{4}, \mathrm{pH} 6.0\right)$ to $60 \% \mathrm{~B}$ solution (30 mM $\mathrm{KH}_{2} \mathrm{PO}_{4}, \mathrm{pH} 6.0$, and $15 \% \mathrm{ACN}$ ) within $15 \mathrm{~min}$, flow rate $1 \mathrm{ml} / \mathrm{min}$ ), and the chromatographic peak at $254 \mathrm{~nm}$ was recorded. The standard solution containing certain concentrations of nucleotides was run in the same HPLC program. Different concentrations of nucleotides were run in the program and calibration curves of $\operatorname{ATP}(0.5-5.0 \mu \mathrm{M}), \operatorname{ADP}(0.5-5 \mu \mathrm{M})$ and AMP $(4.0-15.4 \mu \mathrm{M})$ were generated.

Change in the ATP level following hypoxia treatment was measured by using the ATPLite Adenosine TriPhosphate (ATP) assay kit (PerkinElmer, Boston, MA) following the manufacturer's instruction. Groups of 1-week old male NC or HA flies were collected (10 flies per group) and treated in a $2 \mathrm{kPa} \mathrm{O}$ hypoxic chamber. Flies were collected at different time points during treatment $(0,1,2,5,12$ and 24 hours). Three groups of flies from $\mathrm{NC}$ and HA were analyzed at each time point. The ATP level was normalized against protein level measured in each sample.

\section{Paraquat Treatment and Lifespan}

Paraquat (1,1'-dimethyl-4,4'-bipyridinium dichloride) stock solution $(100 \mathrm{mM})$ was diluted to $5 \mathrm{mM}$ or $10 \mathrm{mM}$ with $5 \%$ sucrose solution for treatment. 5-8 old flies were collected and kept in empty vials for 1 hour prior to treatment. Five vials containing 10 male flies each and 5 vials containing 10 females each were tested for each concentration per experiment. Survival of flies was counted every 24 hours. Freshly prepared PQ solution was added to the vials every 48 hours. The survival curve and $50 \%$ survival rate of each experiment was generated and determined using a sigmoidal nonlinear regression model (GraphPad Prism 4 software, San Diego, CA).

\section{Capillary Feeding Assay}

Daily food intake of $\mathrm{NC}$ and $\mathrm{HA}$ flies was measured in 5-7 days old male flies using a capillary feeding assay as previously described [29]. Briefly, 2 pieces of Whatman filter wetted with $0.5 \mathrm{ml}$ of distilled water was placed at the bottom of a Drosophila Culture vial (Genessee Scientific, San Diego, CA) to provide a water source and moist condition that prevent evaporation of feeding solutions. Flies in groups of 10 were added to the vials and the tube was sealed with Bonded Dense Weave Cellulose Acetate flug which provides an excellent barrier to mite infestation and reduces evaporation (Genessee Scientific, San Diego, CA). A vertical cut on the flug was introduced to hold the capillary feeder. Food was provided by a $75 \mu \mathrm{l}$ micro capillary tube (Warner instrument Corp., Hamden, CT). Capillaries were filled with $25 \mu \mathrm{l}$ of liquid diet (5\% sucrose or apple juice). Fresh food was provided every 24 hours. The height of the liquid column in the capillaries was measured at each food change and for 72 hours. Control experiments were performed to measure the rate of water evaporation under experimental condition using tubes without flies.

\section{Stereological Measurement of Mitochondrial and Myofiber Volume Fractions}

For mitochondrial volume density measurements, eleven TEM images were analyzed for the NG and 15 TEM images for the HA as follows. A $9 \times 13$ rectangular grid (chosen for ease of use with Photoshop) was overlaid on each image, and mitochondria and cytoplasm lying under intercepts were counted. The relative volume density (volume fraction) of mitochondria was expressed as the ratio of intercepts coinciding with this organelle relative to the intercepts coinciding with cytoplasm+mitochondria (including the myofibers) and reported as a percentage. Mitochondrial and myofiber numbers were measured from $6 \mathrm{NC}$ and $10 \mathrm{HA}$ images and normalized to the cross-sectional areas of the cells in each image measured with ImageJ (National Institutes of Health). The cross-sectional area of 69 myofiber bundles was measured from the TEM images.

\section{Electron Microscopy and Tomography}

For electron tomography, sections were cut from the same blocks used for conventional transmission electron microscopy at a nominal thickness of $0.5 \mu \mathrm{m}$ and collected on 100:100 clamshell grids. These sections were poststained for $15 \mathrm{~min}$ in a $2 \%$ uranyl acetate solution followed by $15 \mathrm{~min}$ in Sato lead solution. Two sizes of colloidal gold particles, 15 and $20 \mathrm{~nm}$ in diameter, were deposited on opposite sides of the section to serve as fiducial cues. 
For stability in the beam, the section was coated with carbon. For each reconstruction, a single series of images was collected with a JEOL 4000EX intermediate high voltage electron microscope operated at $400 \mathrm{kV}$. The specimens were irradiated for about $30 \mathrm{~min}$ before initiating a tilt series to limit anisotropic specimen thinning during image collection. During data collection, the illumination was held to near parallel beam conditions. Single-tilt series were recorded using a $4 \mathrm{k} \times 4 \mathrm{k}$ slow scan $\mathrm{CCD}$ camera controlled by the program serialEM at 12,000 magnification. Angular increments of $2^{\circ}$ usually from $-60^{\circ}$ to $+60^{\circ}$ about an axis perpendicular to the optical axis of the microscope were achieved using a computer-controlled goniometer to increment accurately the angular steps. The pixel resolution was $1.2 \mathrm{~nm}$. To improve the signal-to-noise ratio, each image was binned down two times by averaging adjacent pixels so that the final pixel resolution was $2.4 \mathrm{~nm}$. The IMOD software package [70] was used for the complete image processing unless there were problems, and then TxBR software was used to refine the alignment and reconstruction [71]. Five $\mathrm{NG}$ and five HA mitochondrial volumes were generated. Volume segmentation was performed by manual tracing in the planes of highest resolution $(X-Y)$ with the program Xvoxtrace [72]. The reconstructions were visualized using Analyze (Mayo Foundation) and the surface-rendering graphics of Synu (National Center for Microscopy and Imaging Research) as described by Perkins et al. [72]. These programs allow one to step through slices of the reconstruction in any orientation and to model and display features of interest in three dimensions.

Measurements of mitochondrial outer and crista membrane surface areas and the number of cristae per mitochondrion were made within the 10 segmented tomographic volumes by the program Synuarea (National Center for Microscopy and Imaging Research, University of California San Diego). For the cristae width measurements, all the mitochondria generated for our 3D analysis were used and a total of 100 cristae per condition (HA long cristae, HA fragmented cristae, and NC cristae) were measured with ImageJ. The cristae membrane was included in the width.

\section{Statistical Analysis}

All values were represented as means \pm SEM. Statistical significance was calculated by ANOVA analysis. Differences were considered significant if $\mathrm{p}<0.05$.

\section{Supporting Information}

Figure S1 Mitochondrial structural analysis for (1) NG flies exposed to $2 \% \mathrm{O}_{2}$ for 1 hour, (2) HA flies exposed to $2 \% \mathrm{O}_{2}$ for 1 hour, (3) HA flies raised in room air for 1 generation, and (4) HA flies raised in room air for 2 generations. (A and $\mathrm{B}$ ) Example mitochondria of the two structural phenotypes observed. (A) Region inside a NG flight muscle mitochondrion with deformed and swollen cristae $(*)$ observed when $\mathrm{NC}$ or HA flies are exposed to $2 \% \mathrm{O}_{2}$ for 1 hour. Whereas commonly observed, this cristae deformation is still the minority; more mitochondria appear like (B) Typical mitochondrion showing the normal morphology of well-ordered and densely packed cristae in NG or HA flight muscle. (C) Typical mitochondrion from HA flies raised in room air for one generation, showing normal morphology with densely packed cristae. (D) Typical mitochondrion from HA flies raised in room air for two generations, again showing normal morphology. Scale $=500 \mathrm{~nm}$ applies to all panels. (E) Mitochondrial volume density. The mitochondrial volume density was significantly higher with the HA flies at room air for the first generation compared to the
NC flies (38\% HA room air, 1 gen. vs. 29\% NC room air-taken from Figure 1; $<<0.01)$. However, by the second generation, there was no statistical difference (34\% HA room air, 2 gen. vs. $29 \%$ NC room air; $p=0.13)$. There was no statistical difference between $\mathrm{HA}$ and NG flies cultured in $2 \% \mathrm{O}_{2}$ for $1 \mathrm{hr}(\mathrm{p}=0.38)$ or compared with HA $4 \% \mathrm{O}_{2}-$ taken from Figure $1(\mathrm{p}=0.17)$ or $\mathrm{NC}$ room air $(\mathrm{p}=0.12)$, respectively. $\mathrm{n}=10$ for all samples. $(\mathrm{F})$ Percentage of mitochondria with subregions of deformed and swollen cristae, as shown in (A). The NG flies cultured in $2 \% \mathrm{O}_{2}$ for $1 \mathrm{hr}$ had significantly more deformed cristae than did the HA flies cultured in $2 \% \mathrm{O}_{2}$ for $1 \mathrm{hr}(* * \mathrm{p}<0.001)$. Interestingly, the HA flies cultured in room air for 2 generations had less deformed cristae compared with the HA flies cultured in room air for 1 generation $(* \mathrm{p}<0.05)$ and was comparable to the $\mathrm{NG}$ room air. $\mathrm{n}=10$ for all samples. Not surprisingly, the HA flies grown in $4 \%$ $\mathrm{O}_{2}$ had significantly more deformed cristae than the $\mathrm{NC}$ room air cristae.

(TIF)

Movie S1 A mitochondrion from NG fly thoracic muscle. Movie shows the three-dimensional details of a medium-sized mitochondrion reconstructed using electron tomography. Clip1: A rapid sequence through 1052.4 nm-thick slices of the tomographic volume. Clip2: Rotations of the surface-rendered volume after segmentation of the inner and outer membranes. The outer membrane is translucent to visualize the cristae. 42 cristae are present. Clip3: Rotation showing only 8 representative cristae after removal of the inner boundary membrane.

(MP4)

Movie S2 A mitochondrion from HA fly thoracic muscle. Movie shows the three-dimensional details of a large mitochondrion reconstructed using electron tomography. Clip1: A rapid sequence through $504.8 \mathrm{~nm}$-thick slices of the tomographic volume. Clip2: Rotations of the surface-rendered volume after segmentation of the inner and outer membranes. The outer membrane is translucent to visualize the cristae. 641 cristae are present, many of which are branched. Clip3: Rotation showing only 11 representative cristae that demonstrate their fragmentation. Clip4: Zoom in and rotation showing 4 adjacent cristae. Clip5: Restoration of all the cristae and partial rotation ending with the slice through the left-hand portion of the surface rendered mitochondrion.

(MP4)

Movie S3 Geotaxis activity of NG flies under room air condition.

(WMV)

Movie S4 Geotaxis activity of HA flies under room air condition.

(WMV)

Movie S5 Geotaxis activity of NG flies under hypoxic condition with $2 \mathrm{kPa} \mathrm{O}_{2}$.

(WMV)

Movie S6 Geotaxis activity of HA flies under hypoxic condition with $2 \mathrm{kPa} \mathrm{O}_{2}$.

(WMV)

\section{Acknowledgments}

We thank Dr. Gabriel G. Haddad for comments and suggestions, and Iain Hartley for technical help. Individuals and resources of The National Center for Microscopy and Imaging Research (NCMIR) were involved in this work. The NCMIR is supported by NIH awards. 


\section{Author Contributions}

Conceived and designed the experiments: DZ GP SQL MHE. Performed the experiments: DZ GP YHH SYY JT MTT JL JX. Analyzed the data:

\section{References}

1. Hochachka PW, Buck LT, Doll CJ, Land SC (1996) Unifying theory of hypoxia tolerance: molecular/metabolic defense and rescue mechanisms for surviving oxygen lack. Proceedings of the National Academy of Sciences of the United States of America 93: 9493-9498.

2. Lynn EG, Lu Z, Minerbi D, Sack MN (2007) The regulation, control, and consequences of mitochondrial oxygen utilization and disposition in the heart and skeletal muscle during hypoxia. Antioxidants \& redox signaling 9: 13531361.

3. Nouette-Gaulain K, Malgat M, Rocher C, Savineau JP, Marthan R, et al. (2005) Time course of differential mitochondrial energy metabolism adaptation to chronic hypoxia in right and left ventricles. Cardiovascular research 66: 132140 .

4. Hagberg H (2004) Mitochondrial impairment in the developing brain after hypoxia-ischemia. Journal of bioenergetics and biomembranes 36: 369-373.

5. Halestrap AP (2009) Mitochondria and reperfusion injury of the heart-a holey death but not beyond salvation. Journal of bioenergetics and biomembranes 41 : $113-121$.

6. Johnston MV, Trescher WH, Ishida A, Nakajima W (2001) Neurobiology of hypoxic-ischemic injury in the developing brain. Pediatric research 49: 735-741.

7. Jones DP (1986) Renal metabolism during normoxia, hypoxia, and ischemic injury. Annual review of physiology 48: 33-50.

8. Lemasters JJ (2007) Modulation of mitochondrial membrane permeability in pathogenesis, autophagy and control of metabolism. Journal of gastroenterology and hepatology 22 Suppl 1: S31-37.

9. Liu X, Hajnoczky G (2011) Altered fusion dynamics underlie unique morphological changes in mitochondria during hypoxia-reoxygenation stress. Cell death and differentiation 18: 1561-1572.

10. Garlid KD, Costa AD, Quinlan CL, Pierre SV, Dos Santos P (2009) Cardioprotective signaling to mitochondria. Journal of molecular and cellular cardiology 46: 858-866.

11. Murphy E, Steenbergen C (2008) Mechanisms underlying acute protection from cardiac ischemia-reperfusion injury. Physiological reviews 88: 581-609.

12. Rubin GM, Yandell MD, Wortman JR, Gabor Miklos GL, Nelson GR, et al. (2000) Comparative genomics of the eukaryotes. Science 287: 2204-2215.

13. Matthews KA, Kaufman TC, Gelbart WM (2005) Research resources for Drosophila: the expanding universe. Nature reviews Genetics 6: 179-193.

14. Venken KJ, Bellen HJ (2005) Emerging technologies for gene manipulation in Drosophila melanogaster. Nature reviews Genetics 6: 167-178.

15. Bier E (2005) Drosophila, the golden bug, emerges as a tool for human genetics. Nature reviews Genetics 6: 9-23.

16. Bier E, Bodmer R (2004) Drosophila, an emerging model for cardiac disease. Gene 342: 1-11.

17. Bilen J, Bonini NM (2005) Drosophila as a model for human neurodegenerative disease. Annual review of genetics 39: 153-171.

18. Hafen E (2004) Cancer, type 2 diabetes, and ageing: news from flies and worms. Swiss medical weekly $134: 711-719$.

19. Jacobs HT, Fernandez-Ayala DJ, Manjiry S, Kemppainen E, Toivonen JM, et al. (2004) Mitochondrial disease in flies. Biochimica et biophysica acta 1659: $190-196$.

20. Anderson PR, Kirby K, Hilliker AJ, Phillips JP (2005) RNAi-mediated suppression of the mitochondrial iron chaperone, frataxin, in Drosophila. Human molecular genetics 14: 3397-3405.

21. Lefai E, Calleja M, Ruiz de Mena I, Lagina AT 3rd, Kaguni LS, et al. (2000) Overexpression of the catalytic subunit of DNA polymerase gamma results in depletion of mitochondrial DNA in Drosophila melanogaster. Molecular \& general genetics : MGG 264: 37-46.

22. Toivonen JM, O'Dell KM, Petit N, Irvine SC, Knight GK, et al. (2001) Technical knockout, a Drosophila model of mitochondrial deafness. Genetics 159: $241-254$

23. Zhou D, Xue J, Chen J, Morcillo P, Lambert JD, et al. (2007) Experimental selection for Drosophila survival in extremely low $\mathrm{O}(2)$ environment. PLoS ONE 2: e490.

24. Zhou D, Udpa N, Gersten M, Visk DW, Bashir A, et al. (2011) Experimental selection of hypoxia-tolerant Drosophila melanogaster. Proceedings of the National Academy of Sciences of the United States of America 108: 2349-2354.

25. Zhou D, Xue J, Lai JC, Schork NJ, White KP, et al. (2008) Mechanisms underlying hypoxia tolerance in Drosophila melanogaster: hairy as a metabolic switch. PLoS genetics 4: e1000221.

26. Feala JD, Coquin L, Zhou D, Haddad GG, Paternostro G, et al. (2009) Metabolism as means for hypoxia adaptation: metabolic profiling and flux balance analysis. BMC systems biology 3: 91 .

27. Castello PR, Drechsel DA, Patel M (2007) Mitochondria are a major source of paraquat-induced reactive oxygen species production in the brain. Journal of biological chemistry 282: 14186-14193.
DZ GP MTT JX. Contributed reagents/materials/analysis tools: DZ GP MHE SQL. Wrote the paper: DZ GP.

28. Cocheme HM, Murphy MP (2008) Complex I is the major site of mitochondrial superoxide production by paraquat. Journal of biological chemistry 283: 17861798.

29. Vigne P, Frelin C (2010) Hypoxia modifies the feeding preferences of Drosophila. Consequences for diet dependent hypoxic survival. BMC physiology 10: 8 .

30. Baar K (2006) Training for endurance and strength: lessons from cell signaling. Medicine and science in sports and exercise 38: 1939-1944.

31. Saltin B, Gollnick PD (1983) Skeletal muscle adaptability: significance for metabolism and performance. In: Peachey LD, Adrian RH, editors. Handbook of physiology. Bethesda, MD: American Physiological Society. p. 631.

32. Hoppeler H, Vogt M (2001) Muscle tissue adaptations to hypoxia. Journal of experimental biology 204: 3133-3139.

33. van Ekeren GJ, Sengers RC, Stadhouders AM (1992) Changes in volume densities and distribution of mitochondria in rat skeletal muscle after chronic hypoxia. International journal of experimental pathology 73: 51-60.

34. Ferretti G (2003) Limiting factors to oxygen transport on Mount Everest 30 years after: a critique of Paolo Cerretelli's contribution to the study of altitude physiology. European journal of applied physiology 90: 344-350.

35. Howald H, Hoppeler H (2003) Performing at extreme altitude: muscle cellular and subcellular adaptations. European journal of applied physiology 90: 360364.

36. Kayser B, Hoppeler H, Claassen H, Cerretelli P (1991) Muscle structure and performance capacity of Himalayan Sherpas. Journal of applied physiology 70 : 1938-1942.

37. Arismendi-Morillo G (2009) Electron microscopy morphology of the mitochondrial network in human cancer. The international journal of biochemistry \& cell biology 41: 2062-2068.

38. Fitzl G, Meyer U, Wassilew G, Welt K (1998) Morphological investigations of the myocardium of cardiomyopathic hamsters during the postnatal development and experimental hypoxia. A quantitative ultrastructural study. Experimental and toxicologic pathology 50: 245-252.

39. Ne'eman Z, Pinson A (1990) Oxygen and extracellular fluid restriction in cultured heart cells: electron microscopy studies. Cardiovascular research 24: 555-559.

40. Zhang XF, Wang YH, Li Q, Zhang XL, Shen JC, et al. (2010) Changes in genioglossus and their association with serum adiponectin levels in rats subjected to chronic intermittent hypoxia. Chinese medical journal 123: 2249-2253.

41. Scalettar BA, Abney JR, Hackenbrock CR (1991) Dynamics, structure, and function are coupled in the mitochondrial matrix. Proceedings of the National Academy of Sciences of the United States of America 88: 8057-8061.

42. Renken C, Siragusa G, Perkins G, Washington L, Nulton J, et al. (2002) A thermodynamic model describing the nature of the crista junction: a structural motif in the mitochondrion. Journal of structural biology 138: 137-144.

43. Cenacchi G, Papa V, Fanin M, Pegoraro E, Angelini C (2011) Comparison of muscle ultrastructure in myasthenia gravis with anti-MuSK and anti-AChR antibodies. Journal of neurology 258: 746-752.

44. Damiano M, Starkov AA, Petri S, Kipiani K, Kiaei M, et al. (2006) Neural mitochondrial Ca2+ capacity impairment precedes the onset of motor symptoms in $\mathrm{G} 93 \mathrm{~A} \mathrm{Cu} / \mathrm{Zn}$-superoxide dismutase mutant mice. Journal of neurochemistry 96: 1349-1361.

45. Darshi M, Mendiola VL, Mackey MR, Murphy AN, Koller A, et al. (2011) ChChd3, an inner mitochondrial membrane protein, is essential for maintaining crista integrity and mitochondrial function. Journal of biological chemistry 286: 2918-2932.

46. Song W, Chen J, Petrilli A, Liot G, Klinglmayr E, et al. (2011) Mutant huntingtin binds the mitochondrial fission GTPase dynamin-related protein-1 and increases its enzymatic activity. Nature medicine 17: 377-382.

47. Bhimji S, Godin DV, McNeill JH (1986) Isoproterenol-induced myocardial ischemic injury in the rabbit: functional and ultrastructural alterations. Acta anatomica 127: 205-211.

48. Neely JR, Feuvray D (1981) Metabolic products and myocardial ischemia. American journal of pathology 102: 282-291.

49. Celotto AM, Frank AC, McGrath SW, Fergestad T, Van Voorhies WA, et al. (2006) Mitochondrial encephalomyopathy in Drosophila. Journal of neuroscience 26: $810-820$

50. Acehan D, Xu Y, Stokes DL, Schlame M (2007) Comparison of lymphoblast mitochondria from normal subjects and patients with Barth syndrome using electron microscopic tomography. Laboratory investigation 87: 40-48.

51. Acehan D, Vaz F, Houtkooper RH, James J, Moore V, et al. (2011) Cardiac and skeletal muscle defects in a mouse model of human Barth syndrome. Journal of biological chemistry 286: 899-908.

52. Wada J, Kanwar YS (1998) Characterization of mammalian translocase of inner mitochondrial membrane (Tim44) isolated from diabetic newborn mouse kidney. Proceedings of the National Academy of Sciences of the United States of America 95: 144-149. 
53. David H, Behrisch D, Vivar Flores OD (1985) Postnatal development of myocardial cells after oxygen deficiency in utero. Pathology, research and practice 179: 370-376.

54. Blair E, Redwood C, Ashrafian H, Oliveira M, Broxholme J, et al. (2001) Mutations in the gamma(2) subunit of AMP-activated protein kinase cause familial hypertrophic cardiomyopathy: evidence for the central role of energy compromise in disease pathogenesis. Human molecular genetics 10: 1215-1220.

55. Calmettes G, Deschodt-Arsac V, Gouspillou G, Miraux S, Muller B, et al. (2010) Improved energy supply regulation in chronic hypoxic mouse counteracts hypoxia-induced altered cardiac energetics. PLoS ONE 5: e9306.

56. Lundby G, Pilegaard H, van Hall G, Sander M, Calbet J, et al. (2003) Oxidative DNA damage and repair in skeletal muscle of humans exposed to high-altitude hypoxia. Toxicology 192: 229-236.

57. Martinelli M, Winterhalder R, Cerretelli P, Howald H, Hoppeler H (1990) Muscle lipofuscin content and satellite cell volume is increased after high altitude exposure in humans. Experientia 46: 672-676.

58. Koechlin C, Maltais F, Saey D, Michaud A, LeBlanc P, et al. (2005) Hypoxaemia enhances peripheral muscle oxidative stress in chronic obstructive pulmonary disease. Thorax 60: 834-841.

59. Anderson EJ, Neufer PD (2006) Type II skeletal myofibers possess unique properties that potentiate mitochondrial $\mathrm{H}(2) \mathrm{O}(2)$ generation. American journal of physiology Cell physiology 290: C844-851.

60. Duranteau J, Chandel NS, Kulisz A, Shao Z, Schumacker PT (1998) Intracellular signaling by reactive oxygen species during hypoxia in cardiomyocytes. Journal of biological chemistry 273: 11619-11624.

61. Vanden Hoek TL, Becker LB, Shao Z, Li C, Schumacker PT (1998) Reactive oxygen species released from mitochondria during brief hypoxia induce preconditioning in cardiomyocytes. Journal of biological chemistry 273: 18092-18098.

62. Costantini P, Petronilli V, Colonna R, Bernardi P (1995) On the effects of paraquat on isolated mitochondria. Evidence that paraquat causes opening of the cyclosporin A-sensitive permeability transition pore synergistically with nitric oxide. Toxicology 99: 77-88.

63. Hirai K, Ikeda K, Wang GY (1992) Paraquat damage of rat liver mitochondria by superoxide production depends on extramitochondrial NADH. Toxicology 72: $1-16$.

64. Tawara T, Fukushima T, Hojo N, Isobe A, Shiwaku K, et al. (1996) Effects of paraquat on mitochondrial electron transport system and catecholamine contents in rat brain. Archives of toxicology 70: 585-589.

65. Ueda T, Hirai K, Ogawa K (1985) Effects of paraquat on the mitochondrial structure and Ca-ATPase activity in rat hepatocytes. Journal of electron microscopy 34: 85-91.

66. Ali SS, Hsiao M, Zhao HW, Dugan LL, Haddad GG, et al. (2012) HypoxiaAdaptation Involves Mitochondrial Metabolic Depression and Decreased ROS Leakage. PLoS ONE 7: e36801.

67. Wicks S, Bain N, Duttaroy A, Hilliker AJ, Phillips JP (2009) Hypoxia rescues early mortality conferred by superoxide dismutase deficiency. Free radical biology \& medicine 46: 176-181.

68. Ganetzky B, Flanagan JR (1978) On the relationship between senescence and age-related changes in two wild-type strains of Drosophila melanogaster. Experimental gerontology 13: 189-196.

69. Klawitter J, Schmitz V, Leibfritz D, Christians U (2007) Development and validation of an assay for the quantification of 11 nucleotides using LC/LCelectrospray ionization-MS. Analytical biochemistry 365: 230-239.

70. Kremer JR, Mastronarde DN, McIntosh JR (1996) Computer visualization of three-dimensional image data using IMOD. Journal of structural biology 116: 71-76.

71. Lawrence A, Bouwer JC, Perkins G, Ellisman MH (2006) Transform-based backprojection for volume reconstruction of large format electron microscope tilt series. Journal of structural biology 154: 144-167.

72. Perkins GA, Renken CW, Song JY, Frey TG, Young SJ, et al. (1997) Electron tomography of large, multicomponent biological structures. Journal of structural biology 120: 219-227. 